\title{
Point-of-care Testing of Coagulation in Intensive Care Unit: Role of Thromboelastography
}

\author{
Deepak Govil ${ }^{1}$, Divya Pal ${ }^{2}$ \\ Indian Journal of Critical Care Medicine (2019): 10.5005/jp-journals-10071-23253
}

\section{INTRODUCTION}

Coagulation and hemostasis is a dynamic process. It is an interplay between primary hemostasis, secondary hemostasis, and fibrinolysis. ${ }^{1}$ Coagulopathy in critically ill patients is common and is of multifactorial origin. Traditionally, the evaluation of disorders of coagulation in critically ill patients is being done using standard laboratory tests that is, prothrombin time with international normalised ratio (PT/INR) and partial thromboplastin time (aPTT). They provide information regarding defect in the extrinsic and intrinsic pathway of coagulation cascade, respectively. These classic tests do not take into account the platelet and vascular interaction contributing to hemostasis and also do not measure the in vivo activity, so the results need to be interpreted carefully.

The use of testing viscoelastic properties of blood allows the measurement of the interaction between the traditional coagulation pathways with platelets, monocytes and fibroblasts and are called global assays of hemostasis. Viscoelastic methods (VEM) have made available the point-of-care testing (POCT) of blood clotting.

\section{Pathophysiology of Coagulopathy in Critically Ill ${ }^{2}$}

Disturbances in basic physiological conditions for hemostasis acidosis, hypocalcemia, hypothermia and hemodilution.

Disturbed primary hemostasis - thrombocytopenia, platelet dysfunction, sepsis or uremia induced derangement, disseminated intravascular coagulation (DIC), drug and heparin-induced thrombocytopenia, massive hemorrhage and multiple blood transfusion.

Blood plasma or clotting factor abnormalities - factor deficiency which could be preexisting or acquired, patients on anticoagulation medication, dysfunction, massive blood loss and multiple blood transfusion.

Hyperfibrinolysis - in major trauma or in postoperative period after major surgeries.

Extracorporeal therapies - due to platelet destruction and heparin use, for example, in cardiac surgeries, extracorporeal membrane oxygenation, ventricular assist devices, renal replacement therapies.

\section{Viscoelastic Methods of Coagulation TESTING ${ }^{3}$}

Viscoelastic methods (VEM) devices are based on the principles of measuring the change in viscoelastic properties of the whole blood during the clot formation. The firmer the clot, the higher is the force that opposes the movement of rotating (in TEG ${ }^{\circledR}$ and ROTEM $^{\circledast}$ ) or vibrating (in Sonoclot) particles of the measuring VEM

\begin{abstract}
1,2 Department of Critical Care Medicine, Medanta: The Medicity, Gurugram, Haryana, India

Corresponding Author: Deepak Govil, Department of Critical Care Medicine, Medanta: The Medicity, Gurugram, Haryana, India, Phone: 91-11-26692531, e-mail: drdeepak_govil@yahoo.co.in

How to cite this article: Govil D, Pal D. Point-of-care Testing of Coagulation in Intensive Care Unit: Role of Thromboelastography. Indian J Crit Care Med 2019;23(Suppl 3):S202-S206.
\end{abstract}

Source of support: Nil

Conflict of interest: None

device. They analyze all phases of coagulation, that is, initiation, amplification, and propagation, and thus reflect the interactions of the cellular and plasma components of coagulation and the activity of the fibrinolytic system. Since they use whole blood, it allows evaluation of the influence of blood cellular components and their phospholipid surfaces on clot formation and its strength.

Different analyzers are available that perform viscoelastic testing including thromboelastography $\left(\right.$ TEG $\left.^{\circledR}\right)$, rotational thromboelastometry $\left(\right.$ ROTEM $\left.^{\circledR}\right)$, Sonoclot analysers.

\section{THROMBOELASTOGRAPHY ${ }^{3}$}

Thromboelastography method was developed by Hartert in 1948. It is a graphic representation of the clotting process, done in the TEG 5000 analyzer (Haemonetics Corporation, Niles, IL, USA; www.haemonetics.com). It provides analysis of platelet function, coagulation proteases and inhibitors, and the fibrinolytic system within 30 minutes, or within 15 minutes if the rapid assay is used.

In classical thromboelastography, $0.36 \mathrm{~mL}$ blood is placed into a heated cuvette or cup which is rotated gently through $4^{\circ}$ $45^{\prime}$ (cycle time $6 / \mathrm{min}$ ) to imitate sluggish venous flow and activate coagulation. Pin is suspended from torsion wire into blood (Fig. 1). Development of fibrin strands "couples" the motion of cup to the pin. "Coupling" is directly proportional to clot strength. Increased tension in wire is detected by electromagnetic transducer. The electrical signal is amplified to create a TEG trace. The result is displayed graphically on pen and ink printer or computer screen. The speed and strength of clot formation is hence measured (Fig. 2) ROTEM $^{\circledast}$ system. $^{3}$

A $0.34 \mathrm{~mL}$ of blood sample, which has been anticoagulated with citrate, is placed into the cuvette, using an electronic pipette. A sensor pin is attached to the shaft, which is connected with a thin spring and slowly oscillates back and forth, suspended in the blood sample. The signal from the pin is transmitted via an optical detector system. As the clot starts to form, this is measured by the ROTEM system and translated to the output in the form of graphical

(0) The Author(s). 2019 Open Access This article is distributed under the terms of the Creative Commons Attribution 4.0 International License (https://creativecommons. org/licenses/by-nc/4.0/), which permits unrestricted use, distribution, and non-commercial reproduction in any medium, provided you give appropriate credit to the original author(s) and the source, provide a link to the Creative Commons license, and indicate if changes were made. The Creative Commons Public Domain Dedication waiver (http://creativecommons.org/publicdomain/zero/1.0/) applies to the data made available in this article, unless otherwise stated. 


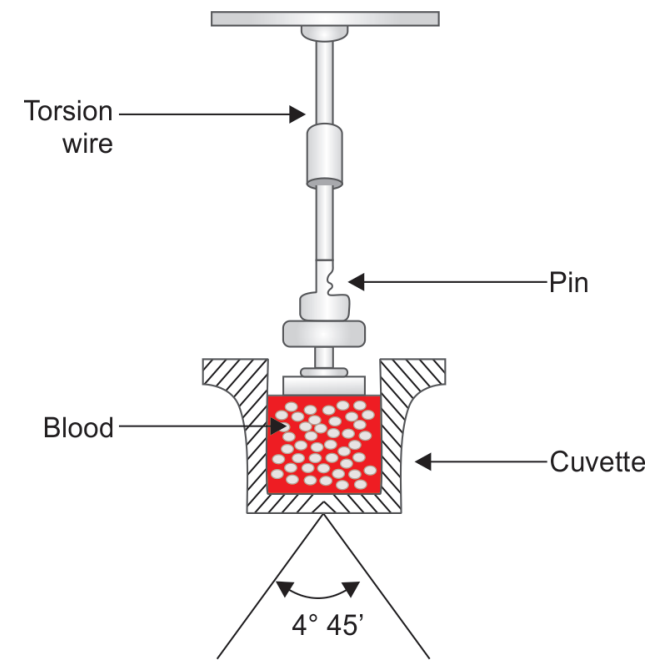

Fig. 1: Thromboelastography technique

display and numerical parameters. The difference between TEG and ROTEM includes the use of torsion wire in TEG rather than the optical detector used in ROTEM to measure the clot formation, and, also that the movement in ROTEM is initiated with the pin while with TEG it is initiated from the cuvette.

The assays used in TEG and ROTEM differ and so the nomenclature is used with the both (Table 1).

\section{Sonoclot ${ }^{\circledR}$ Analyzer ${ }^{3}$}

It is another VEM the process of which is similar to ROTEM and TEG. The Sonoclot analyzer has a hollow open-ended disposable plastic probe mounted on an ultrasonic transducer head. It is immersed to a fixed depth in a cuvette containing $0.40 \mathrm{~mL}$ of blood or plasma and it vibrates vertically at a frequency of $200 \mathrm{~Hz}$. Its free vibration is impeded as the sample clots and the increasing impedance is detected by the electronic circuit driving the probe which is converted to an output signal on a paper chart recorder, reflecting the viscoelastic properties of the clot.

The variables measured by different VEMs have been compared in Table 2.,4 Depending on deficiency or abundance of coagulation factors, platelets or thrombolysins, different TEG tracings are obtained (Fig. 3).

\section{Role in Massive Bleeding and Trauma Patients}

Various causes of coagulopathy in trauma include dilutional coagulopathy, because of intravenous fluid resuscitation, hypothermia, acidosis, polytransfusion, consumption coagulopathy, fibrinolysis and severity of lesion, for example, severe head injury causes release of tissue factor. ${ }^{5}$ It is an independent predictor of mortality necessitating achievement of early hemostasis. VEMs can help delineate the prominent features of trauma-induced coagulopathy (TIC). These can diagnose both hypofibrinogenemia and fibrinolysis. The early administration of tranexamic acid and goal-oriented supplementation of fibrinogen or coagulation factors can decrease the use of blood products, namely freshfrozen plasma.

“European guideline on management of major bleeding and coagulopathy following trauma (fifth edition)" recommend early use of combined traditional laboratory tests or viscoelastic methods to assess and monitor hemostasis (Grade 1C). ${ }^{6}$ The ACS TQIP Massive Transfusion in Trauma Guidelines proposed by the American College of Surgeons in 2013, in the USA, presented the test results obtained by the viscoelastic devices, TEG ${ }^{\circledR} 5000$ and ROTEM ${ }^{\circledast}$, as the standard for transfusion or administration of fresh frozen plasma, cryoprecipitate, platelet concentrate, or antifibrinolytic agents in the treatment strategy for traumatic coagulopathy and hemorrhagic shock. ${ }^{7}$

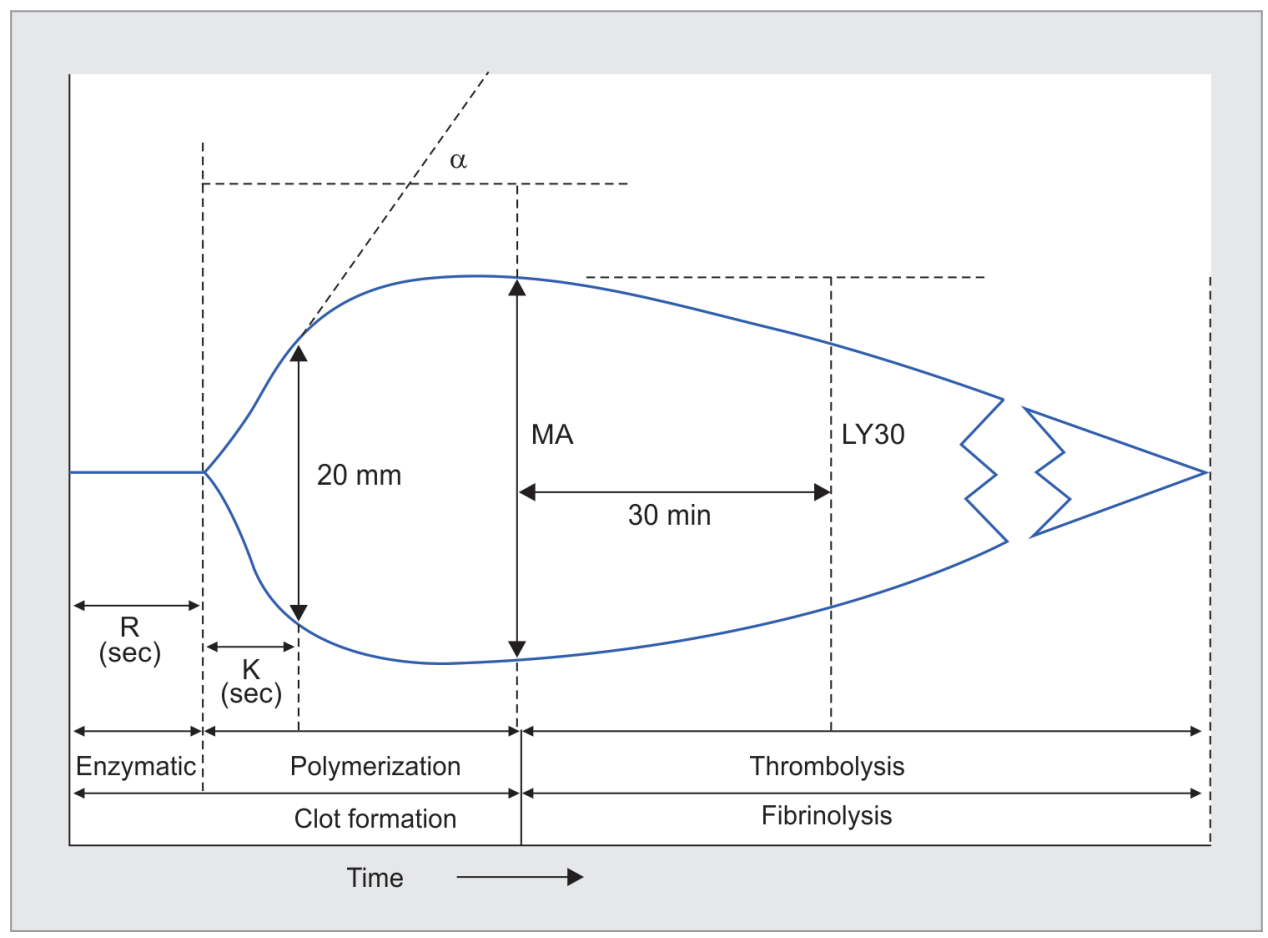

Fig. 2: Thromboelastogram 
Table 1:TEG and ROTEM Assays

\begin{tabular}{|c|c|c|}
\hline Assay & Activator & Interpretation and role \\
\hline \multicolumn{3}{|l|}{ TEG assays } \\
\hline Kaolin & Kaolin & $\begin{array}{l}\text { Assesses clot formation, polymerization, strength and } \\
\text { fibrinolysis. Tests intrinsic pathway }\end{array}$ \\
\hline Heparinase & Kaolin + heparinase & Assesses clot formation in heparinized patients \\
\hline Rapid TEG & Kaolin + tissue factor (TF) & $\begin{array}{l}\text { Provides rapid results compared from standard kaolin } \\
\text { assay. An extrinsic plus intrinsic pathway test. }\end{array}$ \\
\hline Platelet mapping & ADP arachidonic acid & Assesses platelet function \\
\hline Native & None & Nonactivated assay. Native blood clot analysis \\
\hline \multicolumn{3}{|l|}{ ROTEM assays } \\
\hline INTEM & Contact activator- Ellagic acid & $\begin{array}{l}\text { Tests intrinsic pathway. Assesses clot formation, } \\
\text { polymerization, strength and fibrinolysis. }\end{array}$ \\
\hline EXTEM & Tissue factor & Tests extrinsic pathway \\
\hline FIBTEM & $\mathrm{TF}+$ platelet antagonist & Tests fibrin net polymerization after platelet inhibition \\
\hline HEPTEM & Heparinase + contact activator & $\begin{array}{l}\text { Tests residual heparinization. Assesses clot formation in } \\
\text { heparinized patients }\end{array}$ \\
\hline APTEM & TF + Fibrinolysis inhibitor & Tests fibrinolysis \\
\hline NATEM & None & Nonactivated assay. Native blood clot analysis \\
\hline
\end{tabular}

Table 2: Comparison of variables between VEMs and interpretation

\begin{tabular}{|c|c|c|c|c|}
\hline Variable & TEG & ROTEM & Sonoclot & Interpretation \\
\hline $\begin{array}{l}\text { Initial clot formation - } \\
2 \mathrm{~mm} \text { amplitude. Clotting } \\
\text { time }\end{array}$ & $\begin{array}{l}\text { Reaction time- R. Normal- } \\
\text { 3-8 min (citrate/kaolin) }\end{array}$ & Clotting time- CT & $\begin{array}{l}\text { Activated clotting } \\
\text { time- } \mathrm{ACT}\end{array}$ & $\begin{array}{l}\text { Depends on concentration and } \\
\text { function of coagulation factors }\end{array}$ \\
\hline $\begin{array}{l}\text { Clot formation/kinetics - } \\
20 \mathrm{~mm} \text { amplitude } \\
\text { Clot strengthening }\end{array}$ & $\begin{array}{l}\text { Kinetics- K. Normal- 1-3 min. } \\
\text { Alpha angle. Normal- 55-78 } \\
\text { degrees }\end{array}$ & $\begin{array}{l}\text { Clot formation time- } \\
\text { CFT alpha angle }\end{array}$ & Clot rate- CR & $\begin{array}{l}\text { Depends on concentration of } \\
\text { fibrinogen and its activation, also } \\
\text { on platelets to a lesser extent }\end{array}$ \\
\hline $\begin{array}{l}\text { Maximum clot strength/ } \\
\text { firmness }\end{array}$ & $\begin{array}{l}\text { Maximal amplitude- MA. } \\
\text { Normal- 51-69 mm }\end{array}$ & $\begin{array}{l}\text { Maximum clot } \\
\text { firmness- MCF }\end{array}$ & $\begin{array}{l}\text { PEAK-peak } \\
\text { amplitude }\end{array}$ & $\begin{array}{l}\text { Depends on platelet abundance } \\
\text { and function }\end{array}$ \\
\hline Lysis (\%) at fixed time & $\begin{array}{l}\text { Lysis at } 30 \text { and } 60 \text { minutes } \\
\text { post MA- LY30, LY60 }\end{array}$ & $\begin{array}{l}\text { Lysis at } 30,45 \text { and } 60 \\
\text { minutes- LY30, LY45, } \\
\text { LY60 }\end{array}$ & & $\begin{array}{l}\text { Depends on presence of plasmin, } \\
\text { plasminogen and its activators in } \\
\text { blood sample }\end{array}$ \\
\hline
\end{tabular}

Many studies have reported the relationship between the test results obtained by viscoelastic devices and outcome in trauma patients. In a prospective analysis by lves et al., hyperfibrinolysis on thromboelastography developed in approximately $10 \%$ of patients, was considerably more likely to require massive transfusion and was a strong independent predictor of mortality. ${ }^{8}$

In 2014, Yin et al. reported a goal directed transfusion protocol based on the results of TEG ${ }^{\circledR}$ in patients with abdominal trauma. ${ }^{9}$ However, certain limitations of these viscoelastic devices have been reported by some studies. Also, a review in the Cochrane Library published in 2015 pointed out the presence of biases in the studies in trauma patients and the absence of a quality study in this field so far. ${ }^{10}$

\section{Role in Perioperative Period}

The utility of VEMs for managing acute bleeding is well known in cardiac and liver surgeries which are often coupled with coagulopathy of complex origin. There are several factors contributing to bleeding in patients undergoing cardiac bypass surgery like exposure of blood components to extracorporeal circuit, use of heparin and chronic anti platelet medications. Kaolin-Heparinase TEG or HEPTEM assays can be used to assess residual heparinisation effect. Similarly, in the chronic liver disease (CLD) patients, there is mal-production of pro- and anticoagulant factors. The patients undergoing orthotropic liver transplant or

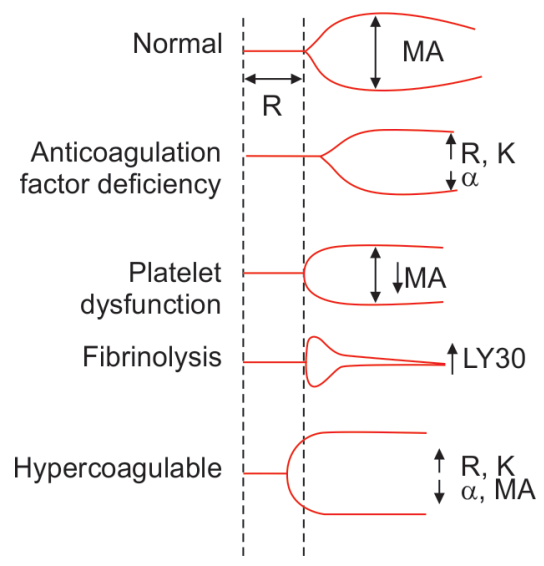

Fig. 3: Thromboelastography tracings - normal and abnormal

hepatectomy can have their blood clotting altered either due to their CLD state, major blood loss or development of acute liver failure in postoperative period or a procoagulant state and a fibrinolytic shutdown. VEM devices can be used to assess the dynamic balance of lysis and secondary hemostasis.

National Institute for Health and Care Excellence (NICE) Diagnostics Guidance 13 reviewed thrombelastography (TEG, ROTEM, and Sonoclot) and concluded that 'The ROTEM system 
and the TEG system are recommended to help detect, manage and monitor hemostasis during and after cardiac surgery', despite methodological problems with the trials. ${ }^{11}$ In a systematic review by Wikkelsoe's group, they identified nine randomized trials (eight cardiosurgical and one orthotopic liver transplantation) which showed the overall benefit of VEM-managed patients in markers like bleeding or blood products use. ${ }^{12}$

In obstetrics, the evidence for use of VEMs is sparse but they can be used to manage bleeding in patients with postpartum hemorrhage which is often associated with consumption coagulopathy and fibrinolysis.

\section{Role in Sepsis and Septic Shock}

Coagulopathy is prevalent in sepsis and occurs because of imbalance between coagulation activation and inhibition and fibrinolysis. This disturbance leads to a variable clinical picture, which ranges from an increased bleeding tendency due to consumption of coagulation factors and platelets, to a hypercoagulable state with disseminated intravascular coagulation (DIC) and (micro-) vascular thrombosis.

In a systematic review by Muller et al., which included 18 studies, they found both hyper- and hypocoagulability and also the TEG/ ROTEM values that fell in the normal range. They concluded that TEG/ROTEM could be a promising tool in diagnosing alterations in coagulation in sepsis. ${ }^{13}$ In some studies patients with septic shock were found to be having a trend toward hypocoagulable state while those without shock had trend toward hypercoagulable state. In an observational cohort study by Adamzik et al., they concluded that the thromboelastometry lysis index was a more reliable biomarker of severe sepsis in critically ill adults compared from procalcitonin, interleukin 6, and C-reactive protein. They also demonstrated early involvement of the hemostatic system as a common event in severe sepsis. ${ }^{14}$

\section{Role in Liver Disease ${ }^{1}$}

The hemostatic changes associated with liver disease affect all aspects of coagulation, that is, primary and secondary hemostasis and fibrinolysis. It is now recognized that these patients cannot be considered 'auto-anticoagulated as systemic hypercoagulability and thrombosis can also be present. Patients with liver disease without decompensation exhibit re-balancing of their haemostatic profile but any trigger may tip the balance towards a bleeding tendency or a pro-thrombotic state. VEMs can help quantify this imbalance and over-aggressive platelet and PT/INR correction may be avoided. Large prospective outcome trials are needed to establish the clinical utility of VEMs in liver disease and to determine threshold values that can predict bleeding or thrombosis and thus help optimise haemostatic therapy.

\section{Hemotherapy Algorithm Including Point-of-Care Techniques for Coagulation Assessment ${ }^{2}$}

Coagulation management in critical care medicine should be based on a hemotherapy algorithm that includes POC techniques. Hemotherapy should involve an assessment of the patient's individual bleeding risk depending on specific situations like major cardiovascular or liver transplant surgery or major trauma. It should also involve evaluation and correction of basic physiological conditions required for haemostasis, for example $\mathrm{pH}$, temperature, serum calcium levels. Repeated evaluation and correction of thrombin and clot formation should also be done. Replacing deficient coagulation factors, if indicated, and improving the hemostatic potential of the primary hemostasis are consecutive steps in therapy escalation. Off-label use of factor XIII and recombinant factor VIla are among the final options.

\section{Advantages of Thromboelastography Testing and Its Clinical Significance ${ }^{4,15}$}

Assesses the blood coagulability globally, that is all phases of coagulation cascade.

- It is a POCT, is rapid and assesses the dynamic changes in blood coagulation.

- Can diagnose coagulopathic bleeding and thus guide goaloriented transfusion therapy and decrease the use of blood products

- It has been shown to be of benefit in detecting a hypercoagulable state in postoperative patients; an increased maximum amplitude (MA or MCF) being the best predictive parameter

- Can predict the clinical efficacy of therapeutic agents affecting blood coagulability

\section{Limitations ${ }^{15}$}

- It measures blood coagulation in vitro, with or without an additional activator.

- The TEG/ ROTEM tracing cannot reflect the contribution of the endothelium to coagulation, so it is poor at detecting conditions that affect platelet adhesion, e.g. von Willebrand's disease.

- It is insensitive to aspirin and clopidogrel and has decreased sensitivity to GPIIb/IIla antagonists.

- The sensitivity and specificity may vary significantly in different populations. For example, in patients on warfarin therapy, Rtime may be normal in TEG with a poor correlation between TEG and INR.

- The device needs daily calibration and test should be performed by trained personnel.

\section{Conclusion}

There is not adequate data available yet regarding the use of POCT for coagulation in critically ill patients in the ICU. Nevertheless, POC coagulation testing is faster and more comprehensive than the traditional laboratory tests and it enables effective and economical management. These POC techniques do not cover the whole spectrum of hemostasis, thus the combination of aggregometric and VEMs is recommended. ${ }^{2}$ Hemotherapy should be based on algorithm that includes POC coagulation testing, which is individualised as per the specific bleeding risks of the patient's condition (e.g., cardiovascular surgery, organ transplantation, and trauma surgery). POC analysis may also enable diagnosis of sepsis-induced hemostatic alterations and may even have the potential to predict mortality in septic and trauma patients.

Finally, prospective randomized trials are needed to analyse whether hemostatic therapy based on POC coagulation testing can be beneficial with respect to clinical outcomes for critically ill patients.

\section{Emerging Indications ${ }^{4}$}

The potential application of TEG to improve the quality of antithrombotic therapy, that is, guiding antiplatelet and anticoagulant 
therapy and to improve the diagnosis, prevention and treatment of venous thromboembolism is a promising avenue for experimental and clinical research.

\section{References}

1. Mallett SV, Chowdhary P, Burroughs AK. Clinical utility of viscoelastic tests off coagulation in patients with liver disease. Liver Int. 2013;33: 961-974.

2. Meybohm P, Zacharowski K, Weber CF. Point-of-care coagulation management in intensive care medicine. Critical Care 2013, 17:218.

3. Whiting P, Al M, Westwood M, Ramos IC, Ryder S, Armstrong N, et al. Viscoelastic point-of-care testing to assist with the diagnosis, management and monitoring of haemostats: a systematic review and cost-effectiveness analysis. Health Technology Assessment 2015;19(58).

4. Shaydakov ME, Blebea J. Thromboelastography (TEG). StatPearls Jan 2019.

5. Galvez K, Cortes C. Thromboelastography: New concepts in haemostasis physiology and correlation with trauma associated coagulopathy. Rev Colomb Anestesiol 2012;40:224-230.

6. Spahn DR, Bouillon B, Cerny V, Duranteau J, Filipescu D, Hunt BJ, et al. European guideline on management of major bleeding and coagulopathy following trauma: fifth edition. Critical Care 2019;23:98.

7. Camazine MN, Hemmila MR, Leonard JC, Jacobs RA, Horst JA, Kozar RA, et al. Massive transfusion policies at trauma centers participating in the American College of Surgeons Trauma Quality Improvement Program. J Trauma Acute Care Surg. 2015;78:S48-53.
8. Ives $\mathrm{C}$, Inaba $\mathrm{K}$, Branco BC, Okoye $\mathrm{O}$, Schochl H, Talving $\mathrm{P}$, et al. Hyperfibrinolysis Elicited via Thromboelastography Predicts Mortality in Trauma. J Am Coll Surg 2012;215:496-502.

9. Yin J, Zhao Z, Li Y, Wang J, Yao D, Zhang S, et al. Goal-directed transfusion protocol via thrombelastography in patients with abdominal trauma: a retrospective study. World J Emerg Surg. 2014;9:28.

10. Hunt H, Stanworth S, Curry N, Woolley T, Cooper C, Ukoumunne O, et al. Thromboelastography (TEG) and rotational thromboelastometry (ROTEM) for trauma induced coagulopathy in adult trauma patients with bleeding. Cochrane Database Syst Rev. 2015;2:CD010438

11. National Institute for Clinical Excellence (NICE). Detecting, managing and monitoring haemostasis: viscoelastometric point- of-care testing (ROTEM, TEG and Sonoclot systems). 2014. Diagnostics Guidance 13.

12. Wikkelsoe AJ, Afshari A, Wetterslev J, Brok J, Moeller AM. Monitoring patients at risk of massive transfusion with thrombelastography or thromboelastometry: a systematic review. Acta Anaesthesiol Scand (2011) 55(10):1174-1189.

13. Muller M, Meijers JC, Vroom MB, Juffermans NP. Utility of thromboelastography and/or thromboelastometry in adults with sepsis: a systematic review. Critical Care 2014;18:R30.

14. Adamzik M, Eggmann M, Frey UH, Gorlinger K, Brocker-Preuss $M$, Marggraf $G$, et al. Comparison of thromboelastometry with procalcitonin, interleukin 6, and C-reactive protein as diagnostic tests for severe sepsis in critically ill adults. Critical Care 2010;14:R178.

15. Srivastava A, Kelleher A. Point-of-care coagulation testing. Continuing Education in Anaesthesia, Critical Care \& Pain 2013;13(1). 